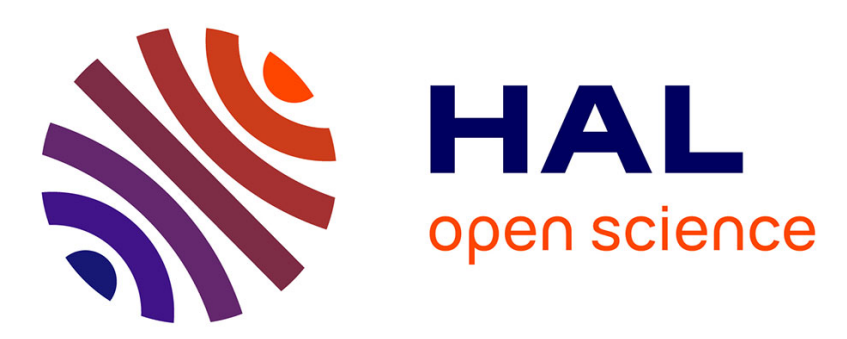

\title{
Application du contrôle optimal à l'identification d'un chargement thermique
}

\author{
Benoît Delattre, Damien Ivaldi, Claude Stolz
}

\section{To cite this version:}

Benoît Delattre, Damien Ivaldi, Claude Stolz. Application du contrôle optimal à l'identification d'un chargement thermique. Revue Européenne des Éléments Finis, 2002, 11, pp.393-404. 10.3166/reef.11.393-404 . hal-00111352

\section{HAL Id: hal-00111352 \\ https://hal.science/hal-00111352}

Submitted on 1 Aug 2019

HAL is a multi-disciplinary open access archive for the deposit and dissemination of scientific research documents, whether they are published or not. The documents may come from teaching and research institutions in France or abroad, or from public or private research centers.
L'archive ouverte pluridisciplinaire HAL, est destinée au dépôt et à la diffusion de documents scientifiques de niveau recherche, publiés ou non, émanant des établissements d'enseignement et de recherche français ou étrangers, des laboratoires publics ou privés. 


\title{
Application du contrôle optimal à l'identification d'un chargement thermique
}

\author{
Benoit Delattre* - Damien Ivaldi** - Claude Stolz \\ * Laboratoire de Mécanique des Solides \\ CNRS UMR 7649 \\ Ecole Polytechnique \\ 91128 Palaiseau cedex \\ \{delattre,stolz\}@lms.polytechnique.fr \\ ** PSA PEUGEOT CITROËN \\ DINQ / DRIA / SARA /MATE \\ Centre de Vélizy - route de Gisy \\ 78943 Vélizy-Villacoublay \\ damien.ivaldi@mpsa.com
}

RÉSUMÉ. La détermination du champ de température sur certaines pièces d'un moteur est obtenue par la résolution d'un problème inverse. Une alternative aux méthodes expérimentales coûteuses utilisées aujourd'hui est la méthode du contrôle optimal qui permet d'estimer le champ de température à partir de quantités mesurées sur pièce. Différents tests préfigurent des possibilités de prédiction et de fiabilité de la méthode.

ABSTRACT. We are faced with an inverse problem in the determination of some engine temperature concerning unreacheable pieces. To reduce the experimental costs, PSA tends to develop the theoretical method of optimal control. It allows us to approach the effective temperature using measured physical data and hard- and software possibilities. We collected mathematical and experimental results which provide us a way of evaluating the reliability and accuracy of the method.

MOTS-CLÉs : contrôle optimal, thermique, identification.

KEYWORDS: optimal control, thermal, identification. 


\section{Position du problème}

La conception d'une pièce mécanique dépend de la sollicitation à laquelle elle sera soumise lors de son utilisation en service. La détermination des chargements thermomécaniques de certains de ses éléments peut s'avérer particulièrement difficile. Sur une structure aussi complexe que la culasse d'un moteur automobile, une partie de sa surface extérieure se trouve inaccessible, enfouie au cœur du moteur et par là-même hermétique à toute mesure thermomécanique. Dans de tels cas, nous cherchons une estimation du champ de température appliqué à la face-flamme, partie supérieure de la chambre de combustion, figure 1, pour effectuer ensuite une simulation thermomécanique réaliste en vue de l'estimation de la tenue de la structure. Cette méthode d'identification s'intègre dans la démarche de dimensionnement d'un moteur.

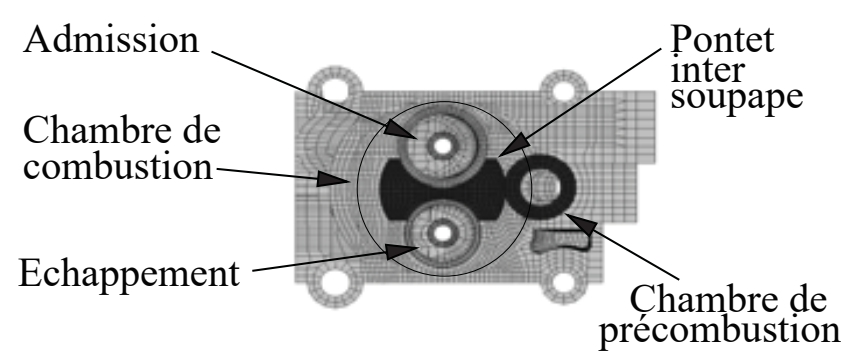

Figure 1. Face-flamme sur une culasse monocylindre

L'identification du chargement thermique de la face-flamme se présente comme un problème mal posé, figure 2, étant dans l'incapacité de mesurer les conditions imposées en température et en flux sur certaines parties de la culasse. Il faut donc élaborer une méthode efficace de reconstruction des conditions de chargement thermique dans un contexte lié à une utilisation industrielle : rapidité et convergence des calculs, utilisation de logiciels standards (dans notre cas : ABAQUS), bon caractère prédictif, etc.

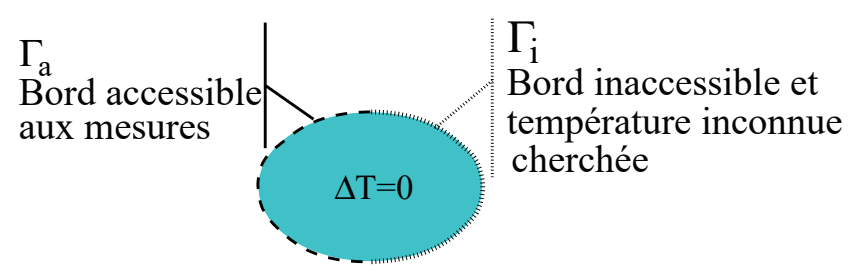

Figure 2. Problème mal posé au sens de Hadamard

Pour cette étude, nous nous restreignons à un calcul thermique linéaire. En vue d'une exploitation ultérieure plus approfondie de la méthode, nous utilisons le logiciel ABAQUS qui permet des calculs non linéaires. Nous effectuons des simulations en deux dimensions sur des géométries simplifiées, principalement une plaque carrée et trouée ainsi qu'une plaque rectangulaire pleine.

Soit un domaine $\Omega$, de frontière $\delta \Omega=\Gamma_{a} U \Gamma_{i}$. Sur $\Gamma_{a}$, les températures, et/ou les flux de chaleur, et/ou des conditions mixtes (loi de convection ...) sont connues 
ou mesurées. Sur $\Gamma_{i}$, on cherche à reconstruire le champ inconnu de température par une méthode d'optimisation. Lors des mesures sur pièces réelles, des thermocouples sont placés à l'intérieur de la pièce, ils apportent des données en températures supplémentaires.

\section{Méthode du contrôle optimal}

\subsection{Méthode générale}

Suivons les méthodes proposées par [BON 00] et [BOU 98]. Nous connaissons la température $T^{0}$ et le flux $q^{0}$ sur le bord $\Gamma_{a}$ par des mesures. La résolution d'un problème thermique avec de telles données aux limites est mal posé.

Pour résoudre la reconstruction du champ, on utilise la résolution de problèmes bien posés auxiliaires. A une distribution de température $v$ sur le bord $\Gamma_{i}$ supposée connue, on note $T\left(v, q^{0}\right)$ la solution du problème direct [1] pour les conditions aux limites imposées $\left(v, q^{0}\right)$ données sur des portions disjointes de frontière. La formulation variationnelle du problème directe ainsi posé [1] est définie à l'aide du lagrangien [2] du système.

$$
\begin{aligned}
& \left\{\begin{aligned}
-k \cdot \Delta T & =0 & & (\Omega) \\
T & =v & & \left(\Gamma_{i}\right) \\
\underline{\nabla T} \cdot \underline{n} & =q^{0} & & \left(\Gamma_{a}\right)
\end{aligned}\right. \\
& L\left(T, T^{*}\right)=\int_{\Omega} \underline{\nabla T} \cdot \underline{\underline{k}} \cdot \underline{\nabla T^{*}} d \omega-\int_{\Gamma_{u}} k \cdot T^{*} \cdot q^{0}
\end{aligned}
$$

Dans cette formulation variationnelle, nous n'utilisons pas simplement un champ $T^{*}$ admissible mais l'état adjoint du système, c'est-à-dire qu'il est solution du problème adjoint au problème [1]. Dans le cas présent, le laplacien étant un opérateur auto-adjoint, $T^{*}$ et $T$ sont solutions de deux problèmes qu'il est possible de résoudre en parallèle.

Pour une distribution $v$ donnée, ce système est bien posé et possède une solution unique. Pour cette solution, la température $T\left(v, q^{0}\right)$ n'est pas identique à $T^{0}$ sur $\Gamma_{a}$. On cherche alors à optimiser le choix de la distribution $v$ afin de réduire l'écart entre la température $T\left(v, q^{0}\right)$ calculée et la température $T^{0}$ mesurée. Cette optimisation est effectuée dans le cadre de la théorie du contrôle optimal, la variable de contrôle $v$ est déterminée pour rendre minimale une fonction coût.

Le contrôle optimal consiste à construire une fonctionnelle $J\left(T, T^{*}\right)$ composée du lagrangien $L\left(T, T^{*}\right)$ auquel on ajoute des termes de contrôle $Q\left(T, T^{*}\right)$. Ces contrôles sont choisis de manière à rendre le problème bien posé au sens de Hadamard. Dès lors, cette fonctionnelle pourra être minimisée par un logiciel de calcul. Outre un terme de contrôle minimisant l'écart entre $T$ et $T^{0}$ sur le bord $\Gamma_{a}$, on peut introduire des termes 
de contrôle régularisant la température sur le bord $\Gamma_{i}$. Cela nous donne la fonctionnelle [3] qui, une fois minimisée, est équivalente au système [4].

$$
\begin{aligned}
& J\left(T, T^{*}\right)=L\left(T, T^{*}\right) \quad+\int_{\Gamma_{a}} \frac{M}{2}\left(T-T^{0}\right)^{2} d s \quad+\int_{\Gamma_{i}} \frac{r}{2} T^{2} d s \\
& T^{*}=0 \quad\left(\Gamma_{i}\right)
\end{aligned}
$$

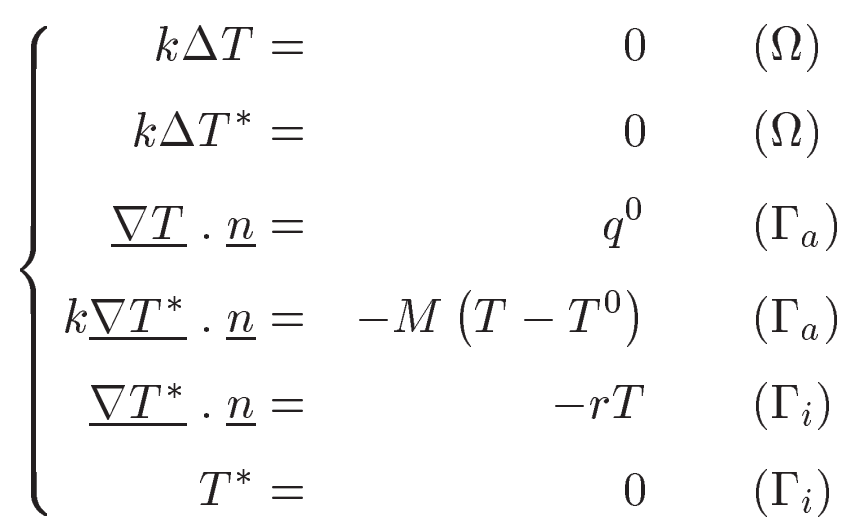

La difficulté principale de la méthode réside dans le choix des termes de contrôle. En particulier, l'unique solution du problème de contrôle dépend du choix des paramètres $M$ et $r$. Les paramètres de contrôle sont choisis de manière à ce que la solution du problème [4] soit une approximation du problème [1]. Pour tester la pertinence des résultats suivant le choix de ces paramètres, nous avons résolu un ensemble de calculs directs où nous imposons la température sur les bords, puis nous utilisons les informations recueillies $\left(\left(T^{0}, q^{0}\right)\right.$ sur $\left.\Gamma_{a}\right)$ comme jeux de données qui permettent l'application de notre méthode.

\subsection{Choix de la fonction coût}

La fonctionnelle $J$ [3] donne de bonnes solutions approchées numériquement par comparaison au calcul direct de référence, mais pose un problème d'ordre pratique. L'écriture du lagrangien nécessite la connaissance du flux de chaleur $q^{0} \operatorname{sur} \Gamma_{a}$. La mesure du flux sur un plan expérimental est peu précise et coûteuse, la fonction coût $J$ n'est pas adaptée pour donner de bons résultats dans un contexte de recherche industrielle. On modifie la fonctionnelle comme suit :

$$
\begin{aligned}
K\left(T, T^{*}\right)= & L\left(T, T^{*}\right)+\sum_{\Omega_{m e s}} \frac{p}{2}\left(T-T_{m e s}^{d}\right)^{2}+\int_{\Gamma_{i}} \frac{r}{2} T^{2} d s \\
& +\int_{\Gamma_{i}} \frac{q}{2}(\underline{\nabla T} \cdot \underline{t})^{2} d s \\
T= & T^{0} \quad\left(\Gamma_{a}\right) \quad T^{*}=0 \quad(\Gamma)
\end{aligned}
$$


Cette fonctionnelle $K$ [5] a l'avantage de ne pas utiliser la connaissance du flux. La température est imposée sur le bord $\Gamma_{a}$ et les mesures de températures prises par thermocouples à l'intérieur même de la pièce, $T_{m e s}^{d}$, sont utilisées.

Le lagrangien est alors modifié comme $L\left(T, T^{*}\right)=\int_{\Omega} \underline{\nabla T} \cdot \underline{\underline{k}} \cdot \underline{\nabla T^{*}} d \omega$.

Le contrôle consiste à minimiser l'écart entre les températures calculées et valeurs mesurées à l'intérieur (terme en $p$ ) ainsi que le flux tangentiel de chaleur sur $\Gamma_{i}$ (terme en $q$ ). Le mauvais conditionnement de la matrice de conductivité rend impossible son inversion par les logiciels standard de résolution. La résolution peut être effectuée par des logiciels spécialisés (comme MATLAB), les résultats obtenus sont excellents mais inapplicables au calcul de structure complexe comme dans le cas d'une culasse de moteur. Pour pallier cette difficulté, on introduit alors dans un code standard une nouvelle régularisation :

$$
\begin{aligned}
C\left(T, T^{*}\right)= & L\left(T, T^{*}\right) \quad+\sum_{\Omega_{m e s}} \frac{p}{2}\left(T-T_{\text {mes }}^{d}\right)^{2}+\int_{\Gamma_{i}} \frac{r}{2} T^{2} d s \\
& +\int_{\Omega} \frac{\varepsilon}{2} k\left[(\nabla T)^{2}+\left(\nabla T^{*}\right)^{2}\right] d \omega \\
T= & T^{0} \quad\left(\Gamma_{a}\right) \quad T^{*}=0 \quad(\Gamma)
\end{aligned}
$$

La fonctionnelle $C$ [6] donne les meilleurs résultats numériques (figure 3 ) en choisissant correctement la valeur de $\varepsilon$ pour améliorer le conditionnement de la matrice à inverser par le contrôle des flux de la température et de la température adjointe sur toute la pièce. De fait, la matrice devient inversible par ABAQUS malgré l'augmentation conséquente de sa largeur de bande. Elle réalise un compromis entre la capacité d'inversion des logiciels et le temps de calcul. Son principal intérêt numérique est de régulariser les valeurs propres de la matrice. De plus, ce terme tend à lisser le champ de température solution.

\subsection{Influence des paramètres de contrôle}

Ces paramètres jouent un rôle important. Faire tendre vers 0 rend l'inversion de la matrice de rigidité impossible. Au contraire, si est d'un facteur mille par rapport aux autres, la solution sera nulle sur quelle que soit la température recherchée. Avec la fonctionnelle [5], le terme dépendant de a tendance à lisser la température sur le bord , ce paramètre doit être minimal pour un chargement thermique très irrégulier sur ce bord. De même, si le coefficient dans la fonctionnelle [6] est prépondérant, la température aura un gradient assez faible. Le coefficient $\mathrm{M}$ quant à lui, améliore la corrélation entre la solution et la température mesurée. Ces coefficients sont donc ajustables en fonction des attentes physiques des résultats. 


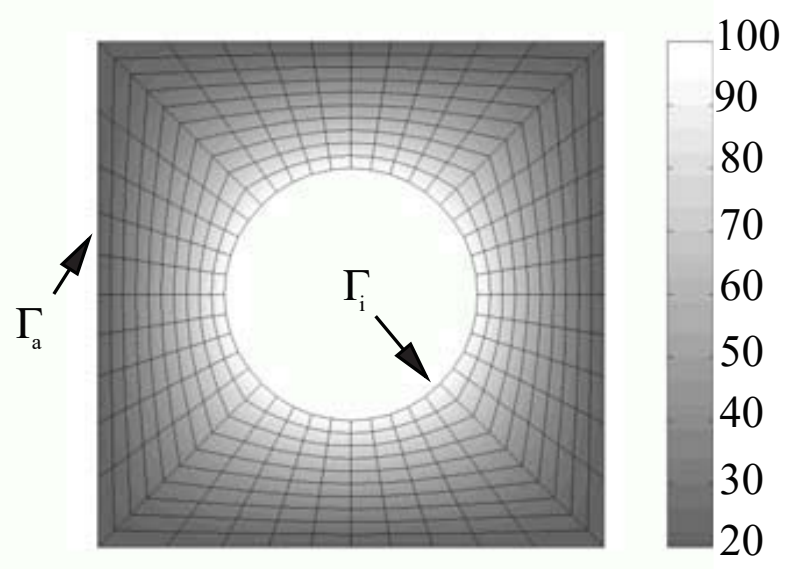

Figure 3. Chargement direct testé. $20^{\circ} \mathrm{C}$ sur $\Gamma_{i}$ et $100^{\circ} \mathrm{C}$ sur $\Gamma_{a}$. La fonctionnelle $C$ retrouve le résultat à $0,05^{\circ} \mathrm{C}$ près

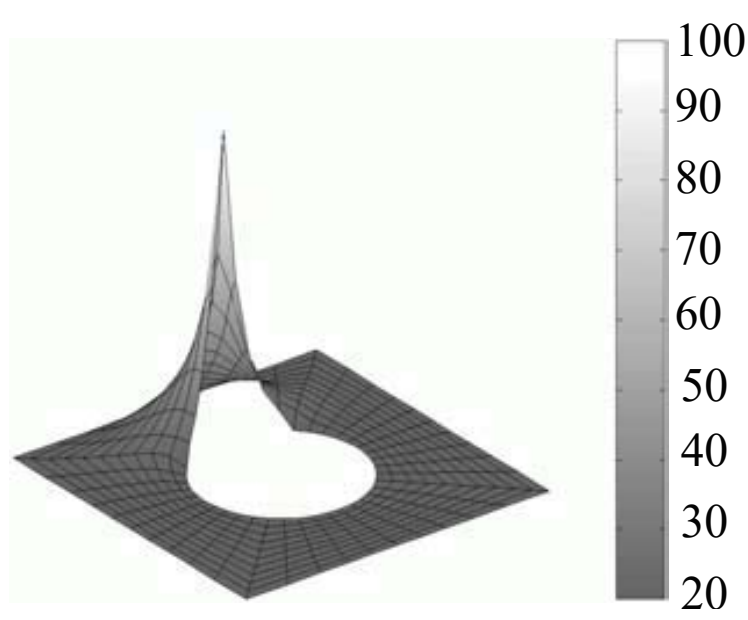

Figure 4. Chargement testé

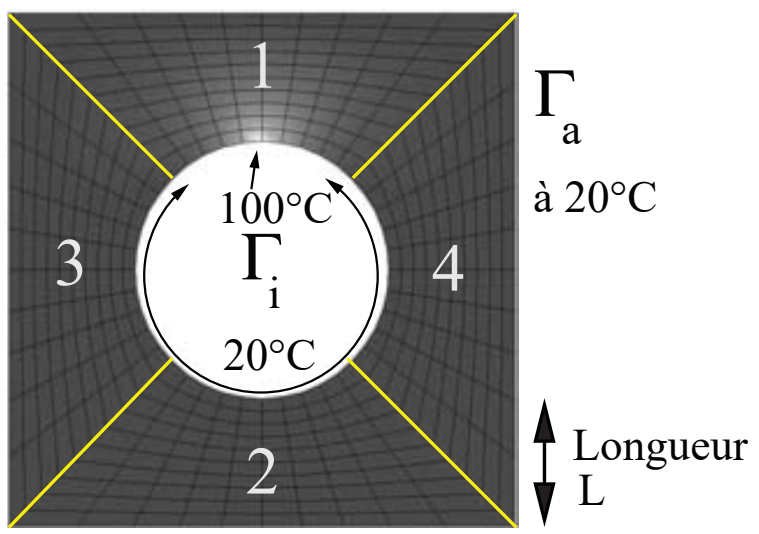

Figure 5. Découpage en zone 


\section{Résultats numériques et expérimentaux}

\subsection{Sensibilité numérique aux termes de contrôle}

Pour tester les limites numériques de la méthode, nous avons envisagé un chargement thermique extrême, figure 4 , avec un fort gradient sur $\Gamma_{i}$. L'idée est de mesurer l'influence du nombre de points de mesure et de leur position sur le champ de température. Nous pouvons distinguer quatre zones d'influence sur la figure 5. En prenant comme référence un ensemble de mesures uniformément réparties dans les zones regroupant au total $4 \%$ de l'ensemble des points du maillage, nous constatons que la zone 2 a une influence négligeable sur la température trouvée au point chaud. Passer d'un volume de points de 0,5 à $3 \%$ dans la zone 2 inflige une erreur supplémentaire de $0,2 \%$ seulement. Les zones 3 et 4 ont une influence double par rapport à la zone 2. Les meilleurs résultats, figure 6 , sont obtenus pour un pourcentage de points de $2 \%$ dans la zone 1 et de $0,5 \%$ dans les autres. La distance moyenne des points au bord $\Gamma_{i}$ est de $2 / 10$ de la longueur. L'erreur entre le chargement réel et le chargement calculé est de $6 \%$.

La position des points de mesure influe bien évidemment sur le résultat. Mettre les points à une distance moyenne supérieure à la moitié de la longueur $L$ implique des erreurs de l'ordre de 50 à $100 \%$. Si cette distance moyenne est inférieure à 3/10, l'erreur est inférieure à $20 \%$.

Nous avons aussi étudié la sensibilité aux erreurs de mesure. Tout d'abord, en introduisant un bruit de moins de $5 \%$ sur les données sur $\Gamma_{a}$, la solution reste inchangée à $0,3 \%$ près. L'erreur commence à devenir perceptible à partir d'un bruit de $10 \%$. Il n'en va pas de même si nous introduisons du bruit sur les données aux points de mesure intérieurs. Un bruit de $1 \%$ sur ces données induit déjà une erreur deux fois plus importante. Pour un bruit de 10\%, l'erreur est multipliée par 3.

\subsection{Banc de mesure plaque-pleine}

Comme première application expérimentale, une plaque pleine, non mince, en alliage d'aluminium AS7G03 a été instrumentée avec 9 thermocouples et a été placée au-dessus d'un chalumeau, figure 7. Le chargement thermique généré par le chalumeau est inconnu. Nous disposons des températures atteintes au niveau des thermocouples en régime stationnaire. De plus, la plaque est fixée sur un socle en acier dont la température reste à peu près constante et égale à $35^{\circ} \mathrm{C}$. La zone de contact est aussi supposée à température constante $\left(35^{\circ} \mathrm{C}\right)$. Nous pouvons appliquer la fonctionnelle [6] à la résolution de ce problème, comme indiqué sur la figure 8 .

Le code ABAQUS nous fournit le résultat présenté à la figure 9. Nous retrouvons globalement la symétrie cylindrique. Les niveaux de températures obtenus corrèlent les relevés expérimentaux avec un maximum à $240^{\circ} \mathrm{C}$. Cette expérience permet une validation du modèle numérique et constitue aussi une première identification du comportement thermomécanique du matériau. 


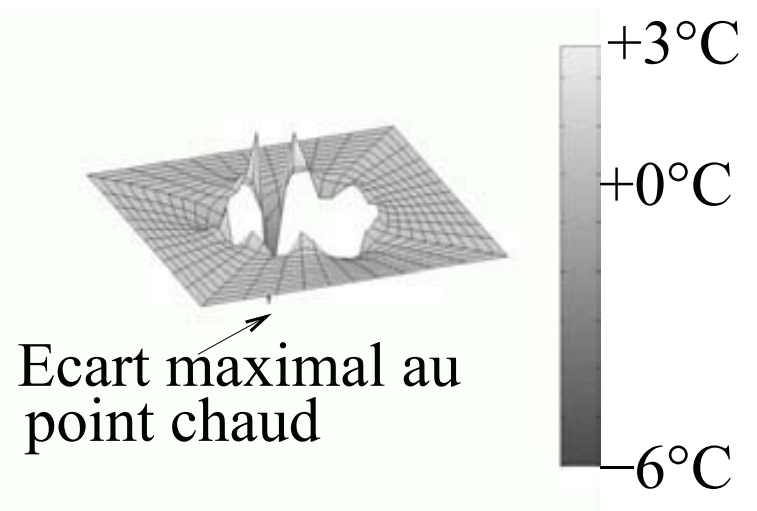

Figure 6. Ecart entre le calcul direct de la figure 4 et le résultat donné par la fonctionnelle $C$

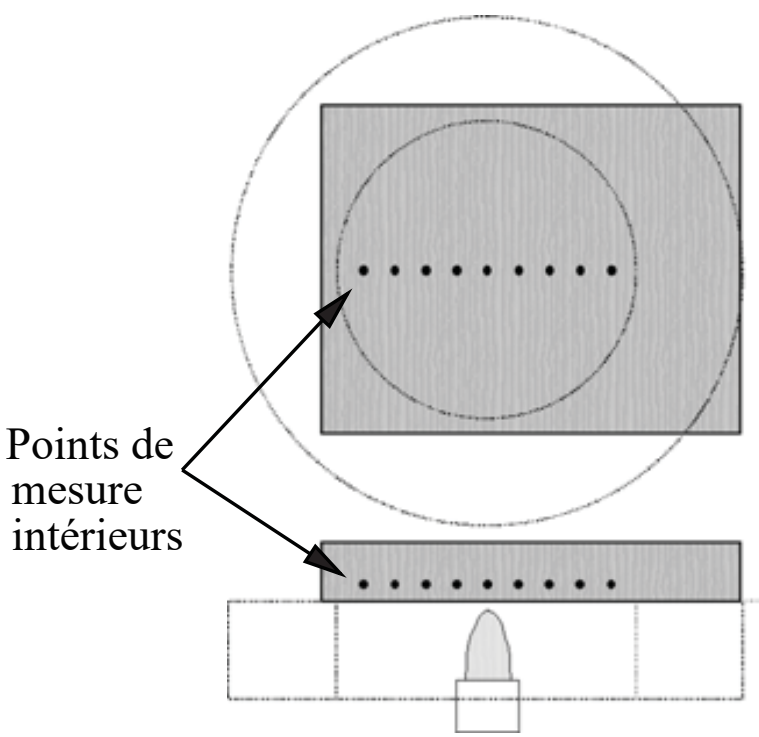

Figure 7. Plaque sur le banc de mesure

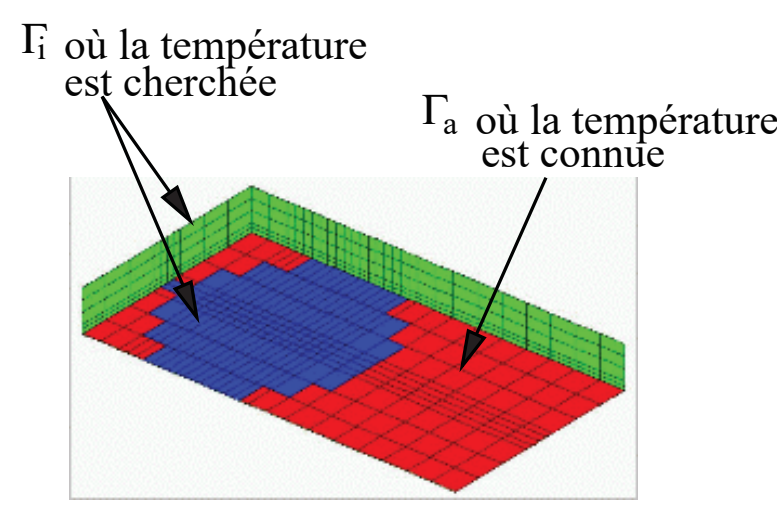

Figure 8. Zones accessibles et inaccessibles 


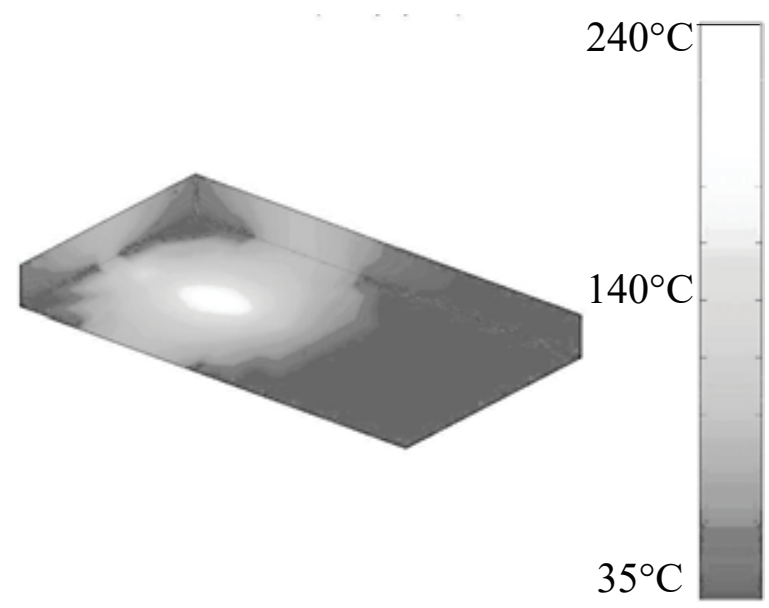

Figure 9. Résultats obtenus par le calcul

\subsection{Banc de mesure face-flamme}

Maintenant, nous instrumentons une autre pièce, une plaque non mince présentant au chalumeau une face (face-flamme) avec deux trous débouchants de la taille des soupapes, figure 10. Pour se rapprocher de la géométrie de la culasse, un noyau d'eau intérieur à la pièce la refroidit en permanence pendant l'expérience. La mesure de la température est double, on dispose de 10 thermocouples (TC) intérieurs et d'une caméra infra-rouge au-dessus de la face opposée à la face-flamme. La plaque est fixée à un socle en acier par des vis. Le chargement thermique imposé lors de l'expérience est cyclique avec une période de chauffage par chalumeau et une période de refroidissement à l'air libre. Seules les températures de régime stationnaire sont utilisées pour l'application de nos fonctionnelles. Ce plan d'expérience va bien au-delà de la validation numérique de notre modèle et permettra à terme de tester l'extension de notre méthode au régime transitoire.

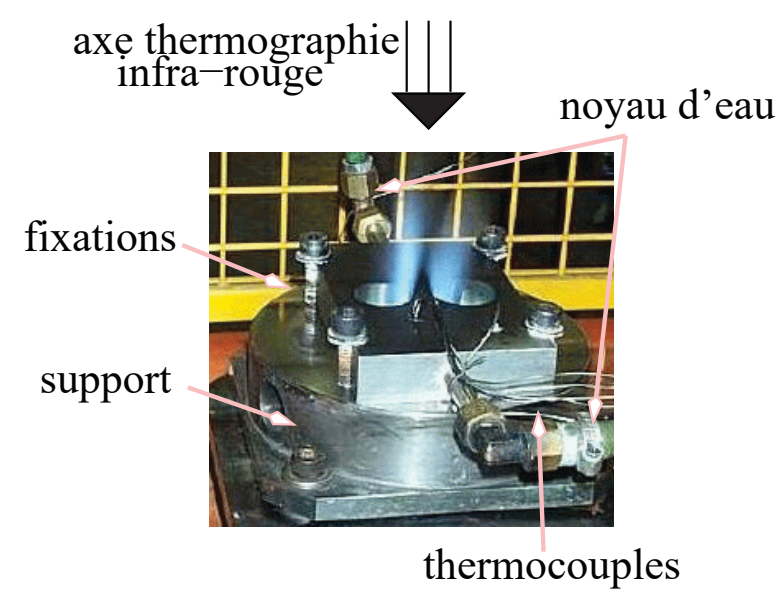

Figure 10. Plaque face-flamme sur le banc de mesure

Pour cette expérience, la fonctionnelle $C$ [6] doit être adaptée. La frontière $\Gamma_{i}$ est décomposée en plusieurs parties. D'abord, elle comprend la face-flamme et les parois intérieures des trous de soupapes. Sur le noyau d'eau, nous pouvons imposer un 


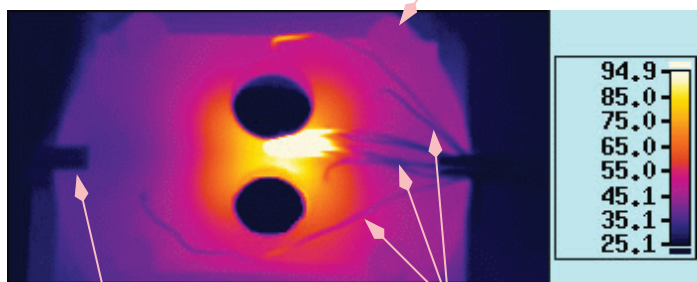

embouts du noyau fils des thermocouples d'eau en surface

Figure 11. Mesure par thermographie infra-rouge

therme de convection thermique avec l'eau injectée, ou choisir de mettre un terme de contrôle en $r$ (dans la pratique, les résultats obtenus avec ces deux options sont équivalents quant à la température sur la face-flamme). Sur la zone de contact (socle et vis), nous pouvons soit imposer une température supposée constante soit contrôler. Les résultats étant là encore équivalents du point de vue de la température utile, nous avons choisi de contrôler. La face offerte à la thermographie infra-rouge (TIR) remplit deux rôles. Les températures observées (figure 11) peuvent être soit un moyen de comparaison avec les températures calculées par contrôle optimal, soit imposées comme condition limite pour raffiner la solution calculée. Les températures données par les TC ainsi que les points symétriques servent de points de mesure (terme en $p$ dans la fonctionnelle). De fait, hormis dans la phase de calcul où on affine les résultats en imposant les températures obtenues par TIR, il n'y a pas de surface $\Gamma_{a}$ dans ce cas précis et nous verrons qu'elle n'est pas nécessaire.

Dans un premier temps, il est nécessaire de déterminer un choix de paramètres optimaux $(r, p, \varepsilon)$. Pour cela, nous utilisons la face de TIR comme partie de $\Gamma_{i}$ et nous comparons le résultat calculé aux températures mesurées. Les deux autres critères employés pour évaluer la validité des températures calculées sont l'écart aux points de mesure et la valeur maximale de la température sur la face-flamme (point "chaud", qui doit être physiquement acceptable). La valeur de référence (égale à 1) des coefficients est donné par celui du lagrangien. Dès lors que $r$ est petit devant 1 (entre $10^{-2}$ et $10^{-5}$ ), ce paramètre n'agit pas sur la qualité de la solution. La valeur de $\varepsilon$, au contraire, a un effet lissage très important : trop petit (moins de $10^{-2}$ ), le champ de température est oscillant en profondeur et en surface, ce qui n'est pas satisfaisant ; trop grand (plus de 1), le point chaud en surface ne dépasse pas le maximum mesuré en profondeur par les TC, de plus l'erreur sur la face TIR est de $100 \%$. L'effet du $p$ est plus complexe. Trop petit (moins de $10^{2}$ ), la solution s'écarte des mesures TC de plus de $25 \%$ et le point chaud du pontet devient froid. Trop grand (plus de $10^{4}$ ), le point chaud devient exagérement chaud (plus de $400^{\circ} \mathrm{C}$ ) et le champ commence à osciller.

Avec ces considérations, nous pouvons trouver un jeu de paramètres optimum mais qui n'est pas encore satisfaisant. En effet, l'écart maximal observé aux TC est encore de $16 \%$ au TC1 (situé au droit du chalumeau) avec l'observation d'un coude dans la température le long du pontet (figure 13) au niveau du TC2, qui est celui placé 


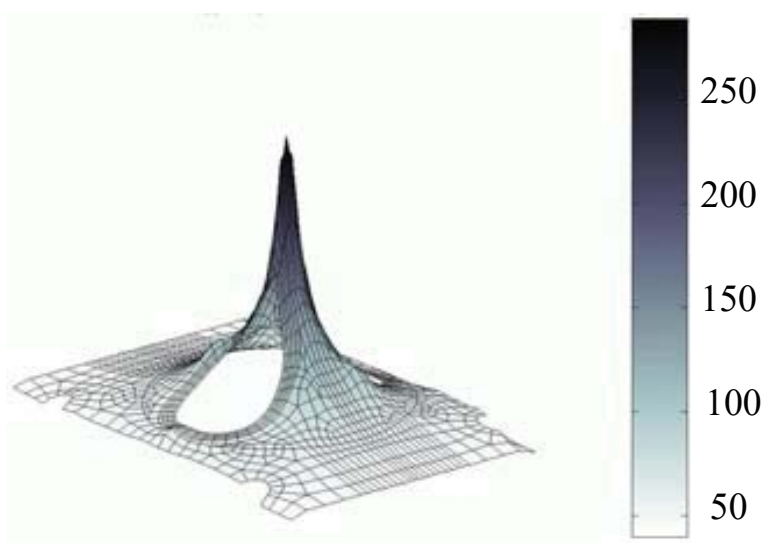

Figure 12. Résultats optimaux calculés sur la face-flamme

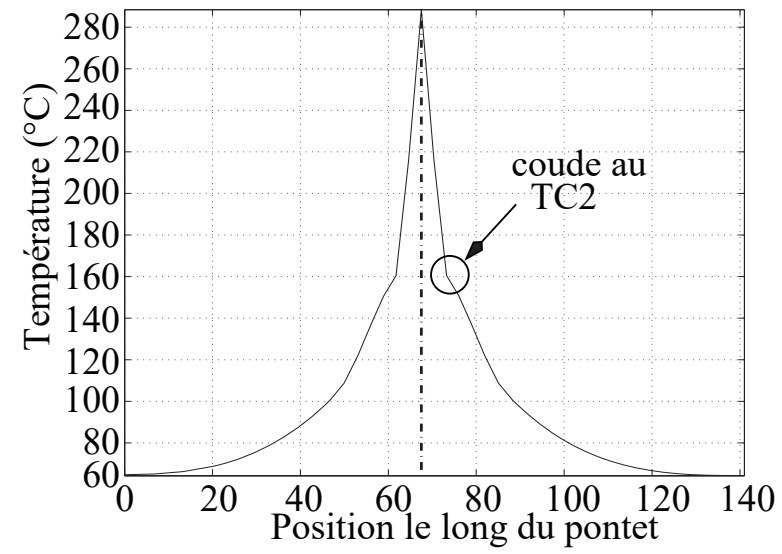

Figure 13. Profil des températures calculées sur l'axe du pontet

au plus près de la flamme après le TC1. Pour reduire ce phénomène tout en gardant les propriétés générales de notre solution, il suffit de prendre deux valeurs différentes pour $p$ : si le $p$ utilisé pour le TC1 est 2 à 3 fois plus grand que celui utilisé pour les autres TC alors on lisse la température observée et on obtient une erreur globale sur les TC de $8 \%$, ce qui se rapproche de l'intervalle de confiance accordé aux mesures. Maintenant, il ne nous reste plus qu'à utiliser ce jeu de paramètres optimal en imposant la température mesurée par TIR sur cette face. Le résultat obtenu reste inchangé sur la partie chaude de la pièce. Seules changent les températures aux bords, ce qui n'influe pas sur le résultat recherché, la température du point chaud. Dans ces conditions, le meilleur champ de température calculé (figures 12 et 13) nous donne un point chaud de $288^{\circ} \mathrm{C}$ pour une erreur maximale de $8 \%$ sur les TC.

La prochaine étape est maintenant d'utiliser les résultats de ces calculs et du TIR pour calculer les flux de chaleur dus au chalumeau et de réinjecter ces informations dans une fonctionnelle adaptée au régime transitoire. 


\section{Conclusion}

La méthode du contrôle optimal présente l'avantage d'une forte adaptabilité. La souplesse trouvée dans le choix des paramètres de contrôle nous permet d'envisager toutes données expérimentales supplémentaires comme contribuant à l'adéquation entre le calcul et la réalité. De plus, il autorise l'opérateur à choisir les données qu'il juge les plus importantes au système. Les paramètres sont d'ailleurs ajustables à la précision des données, pour privilégier les données les plus fiables.

Des études sont en cours pour utiliser cette méthode dans des calculs en contrainte ainsi qu'en régime transitoire.

\section{Bibliographie}

[BON 00] Bonnet M., Problèmes inverses, DEA Dynamique des structures et couplages, Ecole Polytechnique, Paris, 2000.

[BOU 98] Bourgeois L., « Contrôle optimal et problèmes inverses en plasticité », Thèse de doctorat, Ecole Polytechnique, 1998.

[CIA 87] Ciarlet P., The finite element methods for elliptic problems, North-Holland, Amsterdam, 1987.

[CON 94] Constantinescu A., " Sur l'identification des modules élastiques », Thèse de doctorat, Ecole Polytechnique, 1994.

[DEL 00] Delattre B., « Contrôle optimal en analyse thermique », rapport de stage, 2000, PSA.

[DEL 01] Delattre B., IVAldi D., Stolz C., « Application du contrôle optimal à l'identification d'un chargement thermique », Actes du cinquième colloque national en Calcul des structures, Giens, 14-18 mai2001, Technéa, p. 165-172.

[LAR 94] Larrouturou B., Lions P.-L., Méthodes mathématiques pour les sciences de l'ingénieur : Optimisation et analyse numérique, Ecole Polytechnique, Paris, 1994. 